\title{
Infundibulicybe alkaliviolascens (Tricholomataceae): Türkiye Mikotası için Yeni Bir Kayıt
}

\author{
Ertuğrul SESLI ${ }^{1 *}$, Ayşegül TOPCU SESLI ${ }^{2}$ \\ ${ }^{1}$ Karadeniz Teknik Üniversitesi, Fatih Eğitim Fakültesi, Biyoloji Eğitimi Ana Bilim Dalı, \\ Trabzon, Türkiye \\ ${ }^{2}$ IMKB Mesleki ve Teknik Anadolu Lisesi, Yenicuma Mah., Adres Sokak, Ortahisar, \\ Trabzon, Türkiye
}

Öz: Infundibulicybe alkaliviolascens (Bellù) Bellù (Sin.: Clitocybe alkaliviolascens Bellù -Tricholomataceae) Türkiye'den ilk kez rapor edilmiş; arazi ve laboratuvar resimleri ile desteklenip, kısa bir tartışma ile birlikte sunulmuştur.

Anahtar kelimeler: Infundibulicybe alkaliviolascens, Trabzon, Türkiye, Yeni kayıt.

\section{Infundibulicybe alkaliviolascens (Tricholomataceae): A New Record for the Turkish Mycota}

\begin{abstract}
Infundibulicybe alkaliviolascens (Bellù) Bellù (Sin.: Clitocybe alkaliviolascens Bellù - Tricholomataceae) was reported for the first time from Turkey; supported with field and laboratory images and was presented with a short discussion.
\end{abstract}

Key words: Infundibulicybe alkaliviolascens, Trabzon, Turkey, New record.

\section{Giriş}

2003 yılında gerçekleştirilen moleküler çalışmalar ışığında Clitocybe (Fr.) Staude cinsinden ayrılmış olan Infundibulicybe Harmaja, günümüzde dünya çapında 18 türle ve Türkiye'de ise bu çalışmadan önce sadece bir türle (Infundibulicybe geotropa (Bull.) Harmaja) temsil edilen küçük bir cinstir (Staude, 1857; Harmaja, 2003; Sesli ve Denchev, 2008; Solak ve ark., 2015). Cinsin tipik özellikleri olarak, fruktifikasyon organının Clitocybe tipinde olması; bazidiyokarpın, nem oranına göre renk değiştirmemesi; şapkanın tabak veya huni şeklinde, yüzeyinin az çok kadifemsi veya pullu, renginin beyaz, ten renginde, pembemsi soluk kahve, sarımsı kahverengi, kırmızımsı kahverengi veya grimsi kahverengi olması; lamellerin sap üzerine doğru yayılmış; spor izinin beyazımsı ve sporlarının düz ve şeffaf olması; şilosistitlerin bulunmaması; hem toprak ve hem *Sorumlu yazar e-mail: ertugrulsesli@yahoo.com de çimenler üzerinde yayılış göstermesi sayıllabilir (Knudsen ve Vesterholt, 2008).

Son yıllarda Türkiye mikotası ile ilgili olarak gerçekleştirilen çalışmalarda çeşitli yeni kayıtlar saptanmıştır (Kaşık ve ark., 2013; Akata ve ark., 2014; Acar ve ark., 2015; Kaya ve Uzun, 2015; Akata ve ark., 2016; Doğan ve Kurt, 2016; Sesli ve Topcu Sesli, 2016; Topcu Sesli ve Sesli, 2016). Ayrıca, Trabzon ilinden bilim dünyası için bazı yeni cins ve türler tanımlanmıştır (Vizzini ve ark., 2015; Sesli ve ark., 2016; Vizzini ve ark., 2016).

Bu çalışmanın amacı, bazidiyokarpları Trabzon ilinden toplanan Infundibulicybe alkaliviolascens türünü, orijinal resimleri ve kısa bir betimi ile birlikte Türkiye'den ilk kez tanıtmaktır. 


\section{Materyal ve Metot}

Bazidiyokarp örnekleri 2015 yılı Ekim ve Kasım aylarında Karadeniz Teknik Üniversitesi Kanuni ve Fatih yerleşkeleri içerisinde gerçekleştirilen arazi çalışmaları sırasında toplanmıştır. Arazide saptanan bazidiyokarpların öncelikle Canon 600D marka kamera ile fotoğrafları çekilmiştir. Bazidiyokarpların toplandığı alandaki bitki türleri not edildikten sonra örnekler paketlenip mikoloji laboratuvarına getirilmiştir. Spor izleri alındıktan sonra kurutularak fungaryum kutularına yerleştirilen bazidiyokarplardan daha sonra kesitler alınarak mikroskopik yapıları görüntülenmiştir. İncelemelerde Melzer reaktifi, Kongo kırmızısı, potasyum hidroksit, amonyak ve saf su kullanılmıştır. Şapkanın etli kısmına $\mathrm{KOH}$ uygulanmış ve renginin mor menekşeye döndüğü gözlenmiştir. Bazidiyospor, sistityum, bazidiyum, şapka derisi ve hiflerin görüntülenmesinde Zeiss Axio Imager araştırma mikroskopu; ölçümlerin yapılmasında ise Zeiss Axiocam marka kamera ile birlikte Zen 2 yazılım programı kullanılmıştır.

Moleküler çalışmalar için aynı türe ait iki farklı koleksiyondan örnekler alınarak hücrelerin ribozomlarındaki DNA gen dizisine bakılmıştır (ITS). Saptanan gen dizileri Dünya Gen Bankasındaki (http://www.ncbi.nlm.nih. gov /genbank) bu türe ait gen dizisi ile (KR264911) karşılaştırılmış; teşhis hem morfolojik ve hem de moleküler bulgulara göre yapılmıştır.

\section{Bulgular}

Infundibulicybe alkaliviolascens

(Bellù) Bellù, Bresadoliana 1(2): 6 (2012) (Tricholomataceae Lotsy)

[Clitocybe alkaliviolascens Bellù, Beih. Sydowia 10: 29 (1995)] [Türkçe ismi: Pileli] [Şekil 1-3]

Şapka 30-100 mm, soluk sarı, kavun içi, sarımsı kahverengi veya yumurta sarısı; olgunlaşınca özellikle şapka ortasında daha koyu kahverengi, kenarlarda daha açık renklidir. Genç iken meyve tabağı biçiminde, zamanla orta kısmı çukurlaşır ve olgunlukta huni şeklini alır. Kenarları bütün veya parçalı, orta kalınlıkta, olgunlaşınca incelir ve tipik olarak pileli bir görünüm kazanır. Şapkanın etli kısmına $\mathrm{KOH}$ uygulandığı zaman mor menekşe rengine döndüğü görülür. Lameller sık, sarımsı beyaz olup sap üzerine yayılmıştır. Mide bulandırıcı bir kokusu olup tadı hafif yakıcıdır. Sap 40-70 × 5-20 mm, silindirik, genellikle eğri, tabana doğru daha geniş, sarımsı soluk kahverengi, kırmızımsı kahverengi, yer yer tabanda daha yoğun olan beyaz pamuksu yapılarla kaplı, çizgili görünüşte, bazen taban kısmı oldukça şişkindir. Bazidiyosporlar 6-9 × 3.5-5 $\mu \mathrm{m}$, ince çeperli, elips biçiminde, şeffaf, düz veya hafif süslüdür. Bazidiyumlar 25-35 × 6.5-8.5 $\mu \mathrm{m}$, çomak şeklinde, şeffaf, tanecikli ve 4 sporludur. Lamel hifleri düzenli ve kancalı olup sistityum izlenmemiştir.

Türkçe İsim Etimolojisi: "Pileli" ismi, olgunlaşan bazidiyokarpların pileyi anımsatan görünüşünden esinlenilerek verilmiştir.

İncelenen Materyal: Trabzon, Karadeniz Teknik Üniversitesi Fatih Yerleşkesi, 4100'40"K, 39³6'28"D, 20 m, 27 Ekim 2015, gruplar halinde sahil sekoyası altında (Sequoia sempervirens), Fatih Eğitim Fakültesi Kişisel Fungaryumu 3607; Kanuni Yerleşkesi, 4059'39"K, 3946'15"D, 112 m, 2 Kasım 2015; tek tek veya gruplar halinde böğürtlen çalılıkları (Rubus fruticosus) altında, Fatih Eğitim Fakültesi Kişisel Fungaryumu 3645.

\section{Tartışma}

Harmaja (2003) tarafından yapılan moleküler ve morfolojik çalışmalar bazı Clitocybe üyelerinin ayrılarak farklı bir cins olarak sınıflandırılmasının daha uygun olacağı sonucunu ortaya koymuştur. "Infundibulicybe alkaliviolascens" ilk olarak "Clitocybe alkaliviolascens" ismi ile tanınmış; Harmaja (2003) tarafından "Infundibulicybe" adlı bir cins oluşturulduktan sonra, Bellù (2012) tarafından yeni bir kombinasyon olarak bilim dünyasına kazandırılmıştır. 

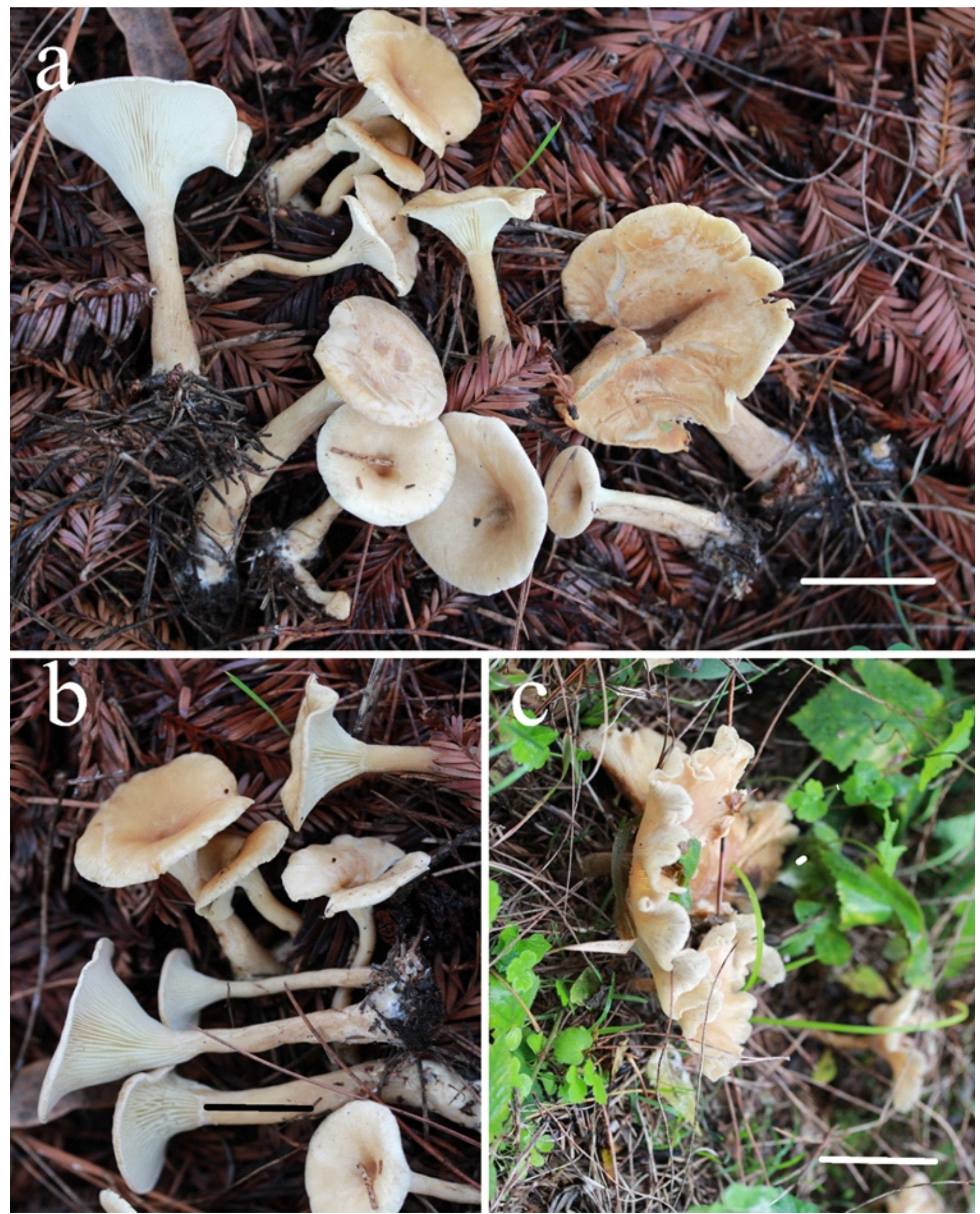

Şekil 1. Infundibulicybe alkaliviolascens: a-c= Bazidiyokarplar (ölçek çubukları: a-c=30 mm). 

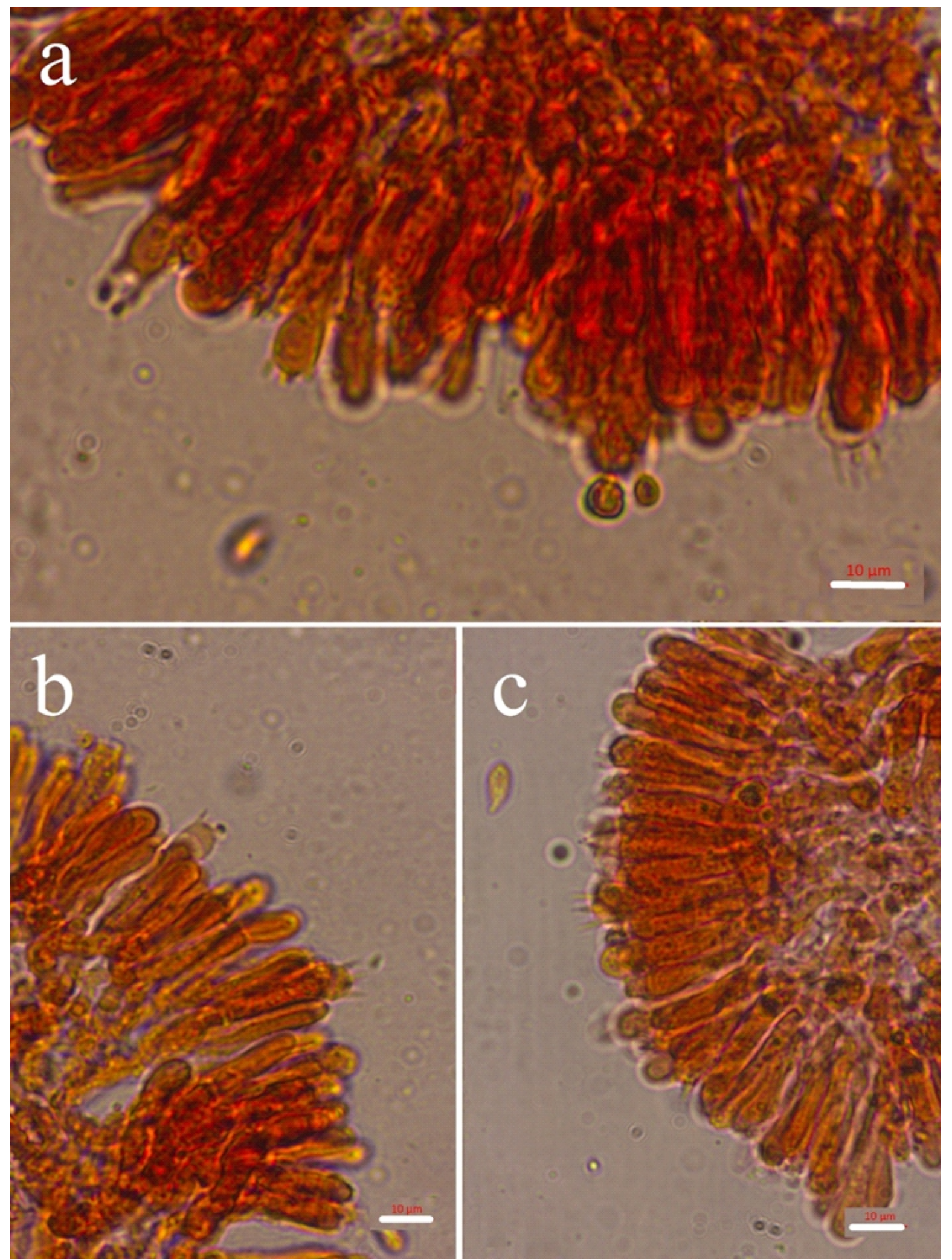

Şekil 2. Infundibulicybe alkaliviolascens: a-c. Bazidiyumlar ve bazidiyoller (ölçek çubukları: a-c= $10 \mu \mathrm{m}$ ). 


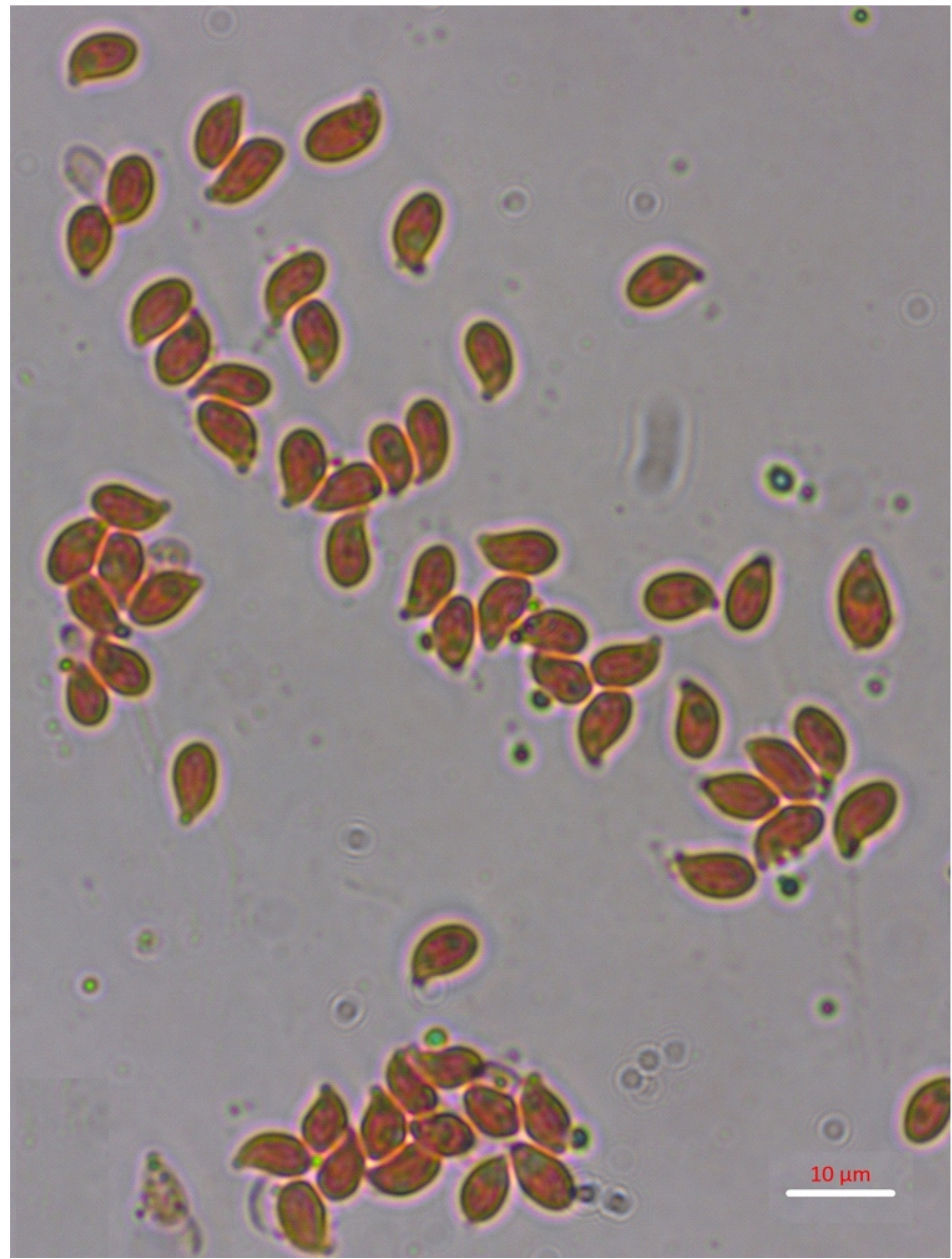

Şekil 3. Infundibulicybe alkaliviolascens: Bazidiyosporlar (ölçek çubuğu: 10 m). 
Mevcut çalışmadan önce, Türkiye'nin çeşitli yörelerinde yapılan çalışmalarda bu cinse ait sadece bir tür (Infundibulicybe geotropa) saptanmıştır (Kaya, 2009; Türkoğlu ve Yağız, 2012).

2015 yılı Sonbaharında Karadeniz Teknik Üniversitesi Kanuni ve Fatih yerleşkeleri içerisinde yaptığımız çalışmalarda söz konusu türe ait iki farklı lokalite tespit edilmiştir.

Tarafımızdan gerçekleştirilen morfolojik çalışmalar; genç bazidiyokarpların soluk sarı veya sarımsı beyaz; şapkanın daha düzgün ve fazlaca bölünmemiş; olgunlaşmış olanların ise koyu sarıdan açık kahverengiye kadar değiştiğini, özellikle şapka kenarlarının pileli bir şekil kazandığını göstermiştir. Mikroskopik çalışmalar, Trabzon örneğinin spor boyutlarının mevcut literatürle tam olarak uyumlu, bazidiyumlarının bir miktar daha uzun olduğunu göstermiştir. Bu durumun ekolojik koşullardan kaynaklanma olasılığın yüksek olduğu sonucuna varılmıştır (Bellù, 2012). Materyalin sekans analiz sonuçları GenBank'taki sonuç ile (KR264911) \%100 oranında benzerlik göstermiştir. Trabzon örneklerine ait sekans analiz sonuçları aşağıdaki gibi saptanmıştır (115917_C7_C7+6525_1F+ITS4):

$\begin{array}{ccc}\mathbf{1} & \text { AACGGGGAAC } & \text { CAACCTGATT } \\ \mathbf{6 1} & \text { TGGACGGTTA } & \text { GAAAGCTGAA } \\ \mathbf{1 2 1} & \text { CCCAATGATG } & \text { GTCAACAATC } \\ \mathbf{1 8 1} & \text { AAGCCCGCAA } & \text { CTCCCATATC } \\ \mathbf{2 4 1} & \text { TTTAATGACA } & \text { CTCAAACAGG } \\ \mathbf{3 0 1} & \text { AGATTCGATG } & \text { ATTCACTGAA } \\ \mathbf{3 6 1} & \text { TCATCGATGC } & \text { GAGAGCCAAG } \\ \mathbf{4 2 1} & \text { CTTTTGACGG } & \text { CGCATTTAAA } \\ \mathbf{4 8 1} & \text { CATAGACTAG } & \text { GGGGAATAAT } \\ \mathbf{5 4 1} & \text { GCTAATGAAA } & \text { GCTTGAAAGT } \\ \mathbf{6 0 1} & \text { AAAATGAGAG } & \text { CAAGCGTGCA } \\ \mathbf{6 6 1} & \text { TTCAATAATG } & \text { ATCCTTCCGC } \\ \mathbf{7 2 1} & \text { CAAATTGGAC } & \text { CAAAGAAAGA }\end{array}$

(114011_D2_D2+6475_1F+ITS4):

1 GGGGGGGAAC CTACCTGATT

61 TGGACGGTTA GAAAGCTGAA

121 CCCAATGATG GTCAACAATC

181 AAGCCCGCAA CTCCCATATC

241 TTTAATGACA CTCAAACAGG

301 AGATTCGATG ATTCACTGAA

361 TCATCGATGC GAGAGCCAAG

421 CTTTTGACGg CGCATTTAAA

481 CATAGACTAG GGgGAATAAT

541 GCTAATGAAA GCTTGAAAGT

601 AAAATGAGAG CAAGCGTGCA

661 TTCAATAATG ATCCTTCCGC

721 CAAATTTGAA CAAAGAAAGG

TGAGGTCAAT
CATTGGCTTC
AAGTTCCACT
CAAGCCAATT
CATGCTCCTC
TTCTGCAATT
AGATCCGTTG
GTTGACATTC
ACAAGGAAAG
GTTCCCAAAA
CATGCCCCAA
AGGTTCACCT
AAAAAAAAA

AGTCAATTAA
ACATCAGAAA
AATGCATTTA
CAATTCAATT
GGAATACCAA
CACATTACTT
TTGAAAGTTG
AAAGACATGC
CGCGCACTAA
GTCTACAATA
AGGGCCAGCG
ACGGAAACCT

ATTATGATTG

TCCAAGTCAA $\mathbf{6 0}$

TGgCGTAGAT AATTATCACA 120

AGGAGAGCCG ACTTGCTGAG $\mathbf{1 8 0}$

CAAATTGAAG AGGTTGAGAA $\mathbf{2 4 0}$

GGAGCGCAAG GTGCGTTCAA $\mathbf{3 0 0}$

ATCGCATTTC GCTGCGTTCT $\mathbf{3 6 0}$

TATTCATTTA TTTTAAAAGG $\mathbf{4 2 0}$

ATTAGgGgTA TATATGAAAA $\mathbf{4 8 0}$

TGCCACATTC CTCAAACCAA $\mathbf{5 4 0}$

AGTGCACAGG TGGTTTGATT $\mathbf{6 0 0}$

ACAACTCACT TCAATTCAAA $\mathbf{6 6 0}$

TGTTACGACT TTTACTTCCT $\mathbf{7 2 0}$

749

TGAGGTCAAT

CATTGGCTTC

AAGTTCCACT

CAAGCCAATT

CATGCTCCTC

TTCTGCAATT

AGATCCGTTG

GTTGACATTC

ACAAGGAAAG

GTTCCCAAAA

CATGCCCCAA

AGGTTCACCT

AAATA
AGTCAATTAA ACATCAGAAA

AATGCATTTA

CAATTCAATT

GGAATACCAA

CACATTACTT

TTGAAAGTTG

AAAGACATGC

CGCGCACTAA

GTCTACAATA

AGGGCCAGCG
ACGGAAACCT
ATTATGATTG

TGGCGTAGAT

AGGAGAGCCG

CAAATTGAAG

GGAGCGCAAG

ATCGCATTTC

TATTCATTTA

ATTAGgGGTA

TGCCACATTC

AGTGCACAGG

ACAACTCACT

TGTTACGACT
TCCAAGTCAA $\mathbf{6 0}$

AATTATCACA $\mathbf{1 2 0}$

ACTTGCTGAG $\mathbf{1 8 0}$

AggtTGaGAA 240

GTGCGTTCAA $\mathbf{3 0 0}$

GCTGCGTTCT $\mathbf{3 6 0}$

TTTTAAAAGG $\mathbf{4 2 0}$

TATATGAAAA $\mathbf{4 8 0}$

CTCAAACCAA $\mathbf{5 4 0}$

TGGTTTGATT $\quad \mathbf{6 0 0}$

TCAATTCAAA $\mathbf{6 6 0}$

TTTACTTCCT $\mathbf{7 2 0}$ 


\section{Teşekkür}

Bu araştırmanın finansmanı Karadeniz Teknik Üniversitesi Bilimsel Araştırma Projeleri Birimince (BAP No: 11300) sağlanmıştır.
Yazının düzenlenmesine yardımcı olan sayın Uzman Ahmet Aydın'a (Karadeniz Teknik Üniversitesi, Fatih Eğitim Fakültesi, İlköğretim Bölümü) içtenlikle teşekkür ederiz.

\section{Kaynaklar}

Acar I., Uzun Y., Demirel K., Keleş A., Macrofungal diversity of Hani (Diyarbakır/Turkey) district, Biological Diversity and Conservation, 8(1)28-34(2015).

Akata I., Kaya A., Uzun Y., Two new Lachnum records for Turkish Mycobiota, Journal of Applied Biological Sciences, 8(1)28-30(2014).

Akata I., Kaya A., Uzun Y., Two new genus records for Turkish Helotiales, Kastamonu Univ. Journal of Forestry Faculty, 16(1)131-134(2016).

Bellù F., Die Trichterlinge der Sekt. Infundibuliformes Fr. und ihre Reaktion gegenüber Kalilauge, Beih. Sydowia, (10)28-34(1995).

Bellù F., Clitocybe alkaliviolascens is now an Infundibulicybe: its recombination with additional distribution data, Bresadoliana, 1(2)3-7(2012).

Doğan H.H., Kurt F., New macrofungi records from Turkey and macrofungal diversity of Pozantı-Adana, Turkish Journal of Botany, (40)209-217(2016).

Harmaja H., Notes on Clitocybe s. Iato (Agaricales), Annales Botanici Fennici, 40(3)213-218(2003).

Kaşık G., Öztürk C., Aktaş S., Alkan S., Eroğlu G., Kefe yaylası (Denizli) yenen mantarları, Mantar Dergisi, 4(2)19-27(2013).

Kaya A., Macromycetes of Kahramanmaraş province (Turkey), Mycotaxon, (108)31-34(2009) + online version: 1-21 (http://www.mycotaxoncom/ resources/checklists/kaya-v108-checklist.pdf).

Kaya A., Uzun Y., Six new genus records for Turkish Pezizales from Gaziantep province, Turkish Journal of Botany, (39)506-511(2015).

Knudsen H., Vesterholt J., Funga Nordica: Agaricoid, Boletoid and Cyphelloid Genera. Narayana Press, Copenhagen(2008).

Sesli E., Denchev C.M., Checklists of the myxomycetes, larger ascomycetes, and larger basidiomycetes in Turkey, Mycotaxon, (106)65-67 (2008). up-dated online version (February 2014): 1-136. http://www.mycotaxon.com/resources/checklists/sesli-v106-checklist.pdf

Sesli E., Topcu Sesli A., Türkiye için üç yeni kayıt: Chalciporus piperatoides, Gymnopus menehune ve Lyophyllum shimeji, Mantar Dergisi, 7(1)61-66(2016).

Sesli E., Vizzzini A., Enrico E., Contu M., Clitolyophyllum akcaabatense gen. nov., sp. nov. (Agaricales, Tricholomatineae), a new fan shaped clitocyboid agaric from Turkey, Botany, (94), 73-80(2016).

Solak M.H., Işıloğlu M., Kalmış E., Allı H., Macrofungi of Turkey.Checklist vol 2.Üniversiteliler Ofset, İzmir(2015).

Staude F., Die Schwämme Mitteldeutschlands, in besondere des Herzogthums, (1)1-150(1857).

Tocu Sesli A., Sesli E., Psilocybe semilanceata (Fries) Kumber(Strophariaceae): Türkiye için yeni bir halüsinojen mantar, Bağbahçe Bilim Dergisi, 3(1)34-40(2016).

Türkoğlu A., Yağız D., Contributions to the macrofungal diversity of Uşak Province, Turkish Journal of Botany, (36)580-589(2012).

Vizzini A., Antonin V., Sesli E., Contu M., Gymnopus trabzonensis sp. nov. (Omphalotaceae) and Tricholoma virgatum var. fulvoumbonatum var. nov. (Tricholomataceae), two new white-spored agarics from Turkey, Phytotaxa, 226(2)119-130(2015).

Vizzini A., Baroni T.J., Sesli E., Antonin V., Saar l.. Rhodocybe tugrulii (Agaricales, Entolomataceae), a new species from Turkey and Estonia based on morphological and molecular data, and a new combination in Clitocella (Entolomataceae), Phytotaxa, 267(1)001-015(2016). 